\title{
Trends in Serum Lipid Profiles Among Korean Adolescents, 2007-2018
}

\author{
Da-Young Jeong' \\ Shin-Hye Kim (D) \\ Moon Young Seo id ${ }^{2}$ \\ Sin Young Kang (D) \\ Mi Jung Park (iD) \\ 'Department of Pediatrics, Inje \\ University Sanggye Paik Hospital, Seoul, \\ Korea; ${ }^{2}$ Department of Pediatrics, \\ Uijeongbu Eulji Medical Center, \\ Gyeonggi-do, Korea
}

\begin{abstract}
Purpose: An adverse lipid profile in adolescence often persists into adulthood, resulting in cardiovascular disease. We aimed to investigate the trends in the prevalence of adverse lipid profiles among Korean adolescents from 2007 to 2018.
\end{abstract}

Patients and Methods: The data were obtained from 9044 adolescents aged 10-19 years who participated in the Korean National Health and Nutrition Examination Surveys (KNHANES) from 2007 to 2018. Fasting serum total cholesterol (TC), high-density lipoprotein cholesterol (HDL-C), low-density lipoprotein cholesterol (LDL-C), and triglyceride (TG) levels were analyzed.

Results: Significantly increasing trends in age-standardized prevalence of hypercholesterolemia [from $5.6 \%$ to $9.4 \%$ in boys $(\mathrm{P}=0.004)$; from $8.1 \%$ to $12.4 \%$ in girls $(\mathrm{P}=0.01)$ ] and hyper-LDL-cholesterolemia [from $4.3 \%$ to $8.0 \%$ in boys $(\mathrm{P}=0.003)$; from $6.6 \%$ to $9.7 \%$ in girls $(\mathrm{P}=0.035)]$ were noted in both sexes. In contrast, the prevalence of hypo-HDLcholesterolemia decreased significantly from $20.0 \%$ to $12.5 \%$ in boys $(<0.001)$ and from $12.4 \%$ to $6.9 \%$ in girls $(\mathrm{P}<0.001)$. There were no significant changes in the prevalence of hypertriglyceridemia and hyper-non-HDL-cholesterolemia in both sexes. Accordingly, the overall prevalence of dyslipidemia showed only a modest decline in boys (from $31.8 \%$ to $28.7 \%$ ) and a stable trend in girls (from $28.9 \%$ to $28.2 \%$ ).

Conclusion: The prevalence of hypercholesterolemia and hyper-LDL-cholesterolemia increased and that of hypo-HDL-cholesterolemia decreased among Korean adolescents over the recent 12 years. Further studies are needed to explain these trends and possible associations with lifestyle factors.

Keywords: hypercholesterolemia, cholesterol, dyslipidemia, prevalence, KNHANES

\section{Introduction}

Atherosclerotic cardiovascular diseases (ASCVD) are the leading causes of morbidity and mortality worldwide. The global prevalence of ASCVD is increasing, and this can be explained by the increasing prevalence of associated risk factors, including obesity, diabetes, and dyslipidemia. ${ }^{1}$ Adverse lipid profile is a major risk factor for ASCVD in adults and has been linked to subclinical atherosclerotic lesions in the pediatric population. ${ }^{2,3}$ Therefore, early detection and management of adverse lipid profiles in youth are crucial from a public health perspective to prevent ASCVD in later life.

In Korea, the prevalence of adverse lipid profiles is expected to increase in parallel to the rising prevalence of obesity in the pediatric population over the past three decades, especially among male adolescents. ${ }^{4}$ The overall prevalence of dyslipidemia in the $2007-2010$ period has been reported to be $21.7-25.2 \%$ in 
Korean teenagers, which is similar to that found among US youths aged 6 to 19 years $(25.4 \%) .{ }^{5,6}$ We previously reported that the prevalence of dyslipidemia in boys was constant between 1998 and 2010 and decreased modestly in girls. ${ }^{7}$ However, no subsequent studies have been conducted on trends in serum lipid profiles in Korean adolescents.

In this study, we aimed to examine the 12-year trends in sex- and age-specific serum lipid concentrations and the prevalence of adverse lipid profiles among Korean adolescents aged 10-19 years in Korea using data from the Korean National Health and Nutrition Examination Surveys (KNHANES) 2007-2018.

\section{Materials and Methods}

\section{Study Population}

The data were obtained from the KNHANES, a survey conducted by The Korea Disease Control and Prevention Agency from 2007 to 2018. A total of 11,581 adolescents aged 10-19 years that participated in the KNHANES 2007-2018 were examined. Adolescents in whom blood lipid profiles were not investigated, those who fasted for less than $9 \mathrm{~h}$ before blood sampling, and those who took medications that could affect the blood glucose and lipid profiles were excluded. The serum low-density lipoprotein cholesterol (LDL-C) levels were calculated from triglyceride (TG) levels using Friedewald's formula. The participants whose TG levels were greater than $400 \mathrm{mg} / \mathrm{dL}$ were also excluded. Finally, 9044 adolescents (4747 boys and 4297 girls) aged 10-19 years were enrolled in this study. (Supplementary Figure 1).

\section{Data Collection and Study Variables}

Participant's height and weight were measured using a stadiometer and a digital weighing scale of $0.1 \mathrm{~cm}$ and $0.1 \mathrm{~kg}$, respectively. The body mass index (BMI) was estimated by dividing the body weight $(\mathrm{kg})$ by the square of the height $(\mathrm{m})$. For participants aged 19 years, overweight and obesity were determined as BMI 23$24.9 \mathrm{~kg} / \mathrm{m}^{2}$ and $\geq 25 \mathrm{~kg} / \mathrm{m}^{2}$, respectively, according to the WHO Western Pacific Region's Asia-Pacific BMI criteria. ${ }^{8}$ For participants aged 10-18 years, overweight and obesity were determined by sex- and age-specific percentile of BMI 85-94.9th percentile and $\geq 95$ th percentile, respectively. ${ }^{9}$ Waist circumference was measured in the standing position at the end of expiration at the middle point of the lower margin of the 12th rib and upper margin of the iliac crest. Central obesity was defined as a waist circumference $\geq 90$ th percentile values for age and sex in participants aged 10-18 years, and waist circumference $\geq 90 \mathrm{~cm}$ for men and $\geq 85 \mathrm{~cm}$ for women in participants aged 19 years based on cutoff points for central obesity in Korean adults. ${ }^{10,11}$

After an overnight fast ( $\geq 9 \mathrm{~h}$ ), fasting blood samples were taken via venipunctures from the antecubital fossa. In 2007, ADIVIA 1650 (Siemens, USA) was used to measure total cholesterol (TC), high-density lipoprotein cholesterol (HDL-C), and TG using an enzymatic colorimeter method. From 2008-2018, Hitachi Automatic Analyzer 7600 (Hitachi, Tokyo, Japan) was used to test TC, HDL-C, and TG levels. Serum LDL-C levels were calculated using Friedewald's formula. ${ }^{12}$

Serum lipid profiles differ by sex and vary with age and pubertal maturation. ${ }^{13}$ As the KNHANES dataset does not include the pubertal stages of the participants, patients were divided into the following age subgroups based on a previous study on lipid profiles of the US pediatric population: $10-11,12-15$, and $16-19$ years. ${ }^{6}$

\section{Definition of Abnormal Lipid Profiles}

In this study, hypercholesterolemia ( $\geq 200 \mathrm{mg} / \mathrm{dL})$, hypertriglyceridemia $(\geq 130 \mathrm{mg} / \mathrm{dL})$, hyper-LDL-cholesterolemia $(\geq 130 \mathrm{mg} / \mathrm{dL})$, hypo-HDL-cholesterolemia $(<40 \mathrm{mg} / \mathrm{dL})$, and hyper-non-HDL-cholesterolemia $(\geq 145 \mathrm{mg} / \mathrm{dL}$ ) were defined according to the NHLBI Expert Panel Guidelines. ${ }^{13}$ The presence of at least one of the following was defined as dyslipidemia: 1) hypercholesterolemia, 2) hypertriglyceridemia, 3) hyper-LDL-cholesterolemia, 4) hypo-HDLcholesterolemia, and 5) hyper-non-HDL-cholesterolemia. ${ }^{14}$

\section{Statistical Analysis}

Data analysis was performed using SPSS version 25.0 for Windows (IBM Inc., Chicago, IL, USA). We performed database configuration, complex multistage, and probability sampling design analyses. Complex samples crosstabs analysis was used for categorical or ordinal variables, and complex samples general linear model analysis was used for continuous variables. We analyzed trends in the ageadjusted prevalence of obesity and adverse lipid profiles through the complex samples general linear model analysis. The concentrations of TGs were log-transformed to obtain a normal distribution. Significant differences were considered at $\mathrm{P}<0.05$ (two-tailed) for all analyses. 


\section{Ethics}

The 2007-2009, 2010-2012, and 2013-2015 KNHANES protocols were approved by the Institutional Review Board (IRB) of the Korea Centers for Disease Control and Prevention (IRB No. 2007-02CON-04-P/200804EXP-01-C/2009-01CON-03-2C， 2010-02CON-21-C/ 2011-02CON-06-C/2012-01EXP-01-2C, and 201307CON-03-4C/2013-12EXP-03-5C). The 2016-2018 KNHANES protocol was exempted from IRB approval based on the Korean bioethics law. Written informed consent was received from all the participants and their parents. Our research protocol was approved by the IRB of Inje University Sanggye Paik Hospital (IRB approval number: SGPAIK 2020-09-013).

\section{Results}

\section{General Characteristics of Participants}

The general characteristics of the study population are described in Supplementary Table 1. The number of subjects was 2645, 2489, 1977, 1933 in KNHANES 20072009, 2010-2012, 2013-2015, 2016-2018, respectively. In boys, significant increases in BMI and waist circumference were observed (both $\mathrm{P}<0.001$ ) over the study period. The prevalence of obesity and central obesity remained unchanged in boys aged 10-11 years, while the prevalence of central obesity significantly increased in boys aged 12 19 years. In girls, no significant changes were observed in the prevalence of obesity or central obesity, although a modest but significant increase in BMI was observed $(\mathrm{P}=0.004)$.

\section{Trends in Mean Values of Serum Lipids}

Trends in age-adjusted mean values of serum lipid profiles are shown in Figure 1, Supplementary Tables 2 and $\underline{3}$. In all participants aged $10-19$ years, age-adjusted TC levels increased from $157.3 \mathrm{mg} / \mathrm{dL}$ to $164.3 \mathrm{mg} / \mathrm{dL}$ in boys and from $162.3 \mathrm{mg} / \mathrm{dL}$ to $168.9 \mathrm{mg} / \mathrm{dL}$ in girls $(\mathrm{P}<0.001)$. The increasing trends were also observed for LDL-C and non-HDL-C, as well as HDL-C in both boys and girls $(\mathrm{P}<0.01)$, (Figure 1) and remained statistically significant after adjusting for BMI and waist circumference (Supplementary Tables 2 and 3 ). However, TG levels showed no significant trend in both sexes.

In sex- and age-specific analyses, a remarkable increase in LDL-C was found in boys aged 10-11 years $(\mathrm{P}=0.042)$ and $16-19$ years $(\mathrm{P}<0.001)$ and in girls aged
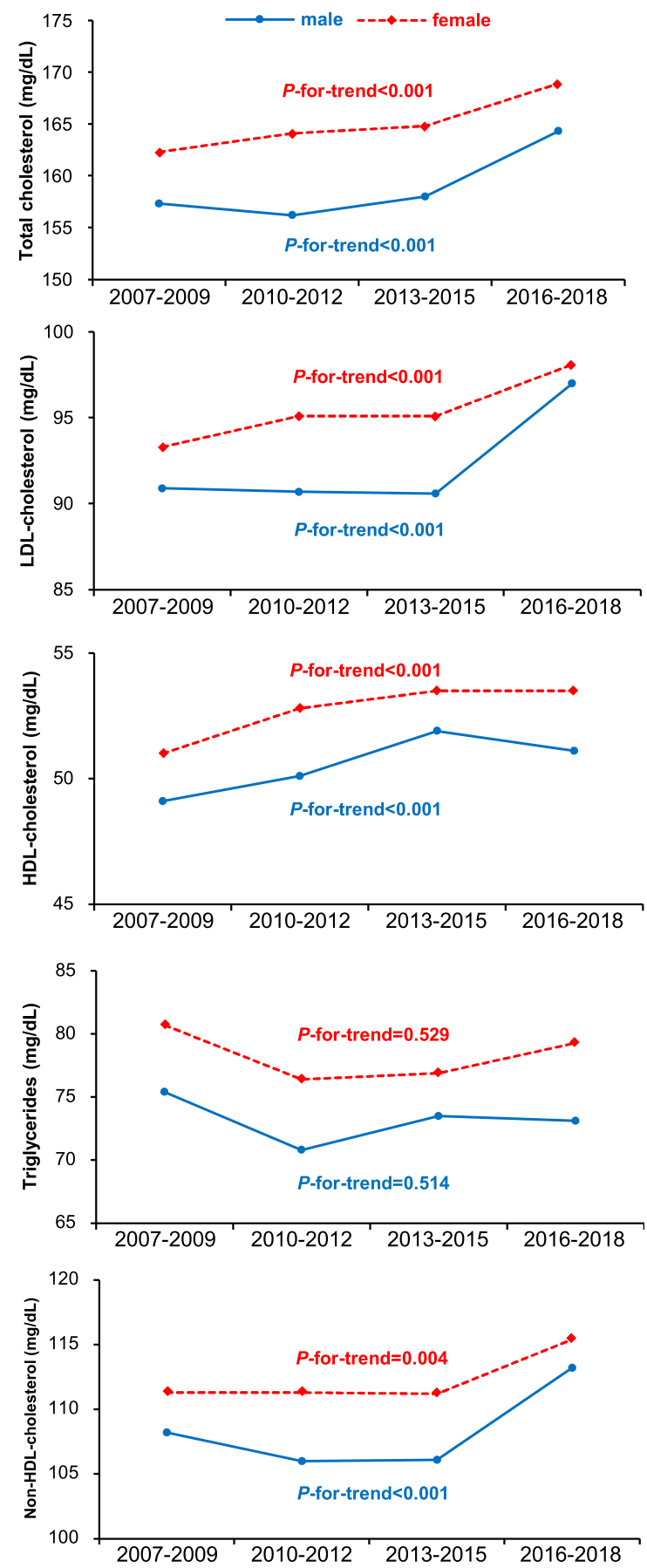

Figure I Trends in age-adjusted mean serum lipid profiles by sex in Korean adolescents from the nationwide survey data from 2007-2018.

Abbreviations: HDL, high-density lipoprotein; LDL, low-density lipoprotein.

12-15 years $(\mathrm{P}=0.037)$ even after adjusting for $\mathrm{BMI}$ and waist circumference (Supplementary Tables 2 and $\underline{3}$ ). A significant increase in HDL-C was observed regardless of age and sex. Increases in both HDL-C and LDL-C contributed to the upward trend of TC in both sexes. The elevation of non-HDL-C was found to be significant only in boys aged $16-19$ years. 
Trends in the Prevalence of Adverse Lipid Profiles

The age-adjusted trends in the prevalence of adverse lipid profiles are presented in Tables 1 and 2. Overall, the prevalence of hypercholesterolemia (from 5.6-8.1\% to 9.4-12.4\%) and hyper-LDL-cholesterolemia (from 4.3$6.6 \%$ to $8.0-9.7 \%$ ) significantly increased in both sexes. In comparison, that of hypo-HDL-cholesterolemia significantly declined in both boys (from $20.0 \%$ to $12.5 \%$ ) and girls (from $12.4 \%$ to $6.9 \%$ ). The prevalence of hyper-nonHDL-cholesterolemia increased (from $8.6-9.4 \%$ to $10.7-$
$12.8 \%$ ) without statistical significance and that of hypertriglyceridemia remained unchanged in both sexes. Since the decline in the prevalence of hypo-HDLcholesterolemia was greater in boys than in girls, the prevalence of dyslipidemia in boys showed a modest but significant decrease (from $31.8 \%$ to $28.7 \%$ ), and the trend in girls remained steady (from $28.9 \%$ to $28.2 \%$ ) (Figure 2).

In sex- and age-specific analyses, the trend in the prevalence of adverse lipid profiles varied across sex and age groups. In boys, an increase in hypercholesterolemia prevalence was observed in boys aged 10-11 years (from

Table I Trends in the Prevalence of Adverse Lipid Profiles by Age in Korean Boys from the Nationwide Survey Data from 2007-2018 $(\mathrm{N}=4747)$

\begin{tabular}{|c|c|c|c|c|c|c|}
\hline \multirow[t]{2}{*}{ Boys } & \multicolumn{4}{|l|}{ Study Year } & \multirow[t]{2}{*}{$P$-for-Trend ${ }^{\mathrm{a}}$} & \multirow[t]{2}{*}{ P-for-Trend } \\
\hline & 2007-2009 & $2010-2012$ & $2013-2015$ & $2016-2018$ & & \\
\hline \multicolumn{7}{|c|}{ Hypercholesterolemia } \\
\hline $10-19$ years & $5.6 \%$ & $5.6 \%$ & $5.3 \%$ & $9.4 \%$ & 0.004 & 0.008 \\
\hline $10-11$ years & $9.8 \%$ & $9.9 \%$ & $8.1 \%$ & $16.3 \%$ & 0.091 & 0.115 \\
\hline $12-15$ years & $4.9 \%$ & $3.9 \%$ & $4.0 \%$ & $5.6 \%$ & 0.659 & 0.786 \\
\hline $16-19$ years & $4.5 \%$ & $5.8 \%$ & $5.1 \%$ & $10.2 \%$ & 0.011 & 0.015 \\
\hline \multicolumn{7}{|c|}{ Hypo-HDL-cholesterolemia } \\
\hline $10-19$ years & $20.0 \%$ & $16.9 \%$ & $10.5 \%$ & $12.5 \%$ & $<0.001$ & $<0.001$ \\
\hline I0-1I years & $11.2 \%$ & $8.3 \%$ & $4.6 \%$ & $9.1 \%$ & $<0.001$ & 0.136 \\
\hline $12-15$ years & $21.6 \%$ & $18.9 \%$ & $11.3 \%$ & $13.2 \%$ & $<0.001$ & $<0.001$ \\
\hline $16-19$ years & $22.0 \%$ & $18.0 \%$ & $12.4 \%$ & $13.8 \%$ & $<0.001$ & 0.001 \\
\hline \multicolumn{7}{|c|}{ Hyper-non-HDL-cholesterolemia } \\
\hline $10-19$ years & $8.6 \%$ & $6.5 \%$ & $6.3 \%$ & $10.7 \%$ & 0.161 & 0.389 \\
\hline I0-1I years & $13.1 \%$ & $8.6 \%$ & $6.7 \%$ & $16.2 \%$ & 0.609 & 0.812 \\
\hline $12-15$ years & $6.7 \%$ & $4.6 \%$ & $4.7 \%$ & $7.1 \%$ & 0.826 & 0.737 \\
\hline $16-19$ years & $8.5 \%$ & $7.8 \%$ & $7.3 \%$ & $11.7 \%$ & 0.192 & 0.261 \\
\hline \multicolumn{7}{|c|}{ Hypertriglyceridemia } \\
\hline $10-19$ years & $14.6 \%$ & $13.1 \%$ & $13.7 \%$ & $12.7 \%$ & 0.357 & 0.118 \\
\hline $10-11$ years & $14.4 \%$ & $10.4 \%$ & $11.2 \%$ & $11.8 \%$ & 0.470 & 0.309 \\
\hline $12-15$ years & $12.9 \%$ & $13.7 \%$ & $13.1 \%$ & $11.5 \%$ & 0.523 & 0.184 \\
\hline $16-19$ years & $16.3 \%$ & $13.6 \%$ & $15.2 \%$ & $14.2 \%$ & 0.621 & 0.459 \\
\hline \multicolumn{7}{|c|}{ Hyper-LDL-cholesterolemia } \\
\hline $10-19$ years & $4.3 \%$ & $5.2 \%$ & $4.9 \%$ & $8.0 \%$ & 0.003 & 0.009 \\
\hline 10-11 years & $6.2 \%$ & $7.6 \%$ & $5.0 \%$ & $13.7 \%$ & 0.029 & 0.047 \\
\hline $12-15$ years & $4.0 \%$ & $3.4 \%$ & $3.5 \%$ & $5.4 \%$ & 0.392 & 0.685 \\
\hline $16-19$ years & $3.9 \%$ & $6.2 \%$ & $5.8 \%$ & $8.2 \%$ & 0.036 & 0.046 \\
\hline \multicolumn{7}{|l|}{ Dyslipidemia } \\
\hline $10-19$ years & $31.8 \%$ & $29.4 \%$ & $24.9 \%$ & $28.7 \%$ & 0.038 & 0.039 \\
\hline $10-11$ years & $28.1 \%$ & $24.8 \%$ & $20.1 \%$ & $34.2 \%$ & 0.419 & 0.419 \\
\hline $12-15$ years & $31.5 \%$ & $30.1 \%$ & $23.5 \%$ & $25.4 \%$ & 0.011 & 0.010 \\
\hline $16-19$ years & $34.1 \%$ & $30.6 \%$ & $27.7 \%$ & $29.5 \%$ & 0.175 & 0.199 \\
\hline
\end{tabular}

Notes: Values are represented as the number (\%). The reference data were obtained from the 2007-2009 nationwide survey. The prevalence was adjusted for age. ${ }^{a}$ Adjusted for age. ${ }^{b}$ Adjusted for age, BMI, and waist circumference.

Abbreviations: HDL, high-density lipoprotein; LDL, low-density lipoprotein. 
Table 2 Trends in the Prevalence of Adverse Lipid Profiles by Age in Korean Girls from the Nationwide Survey Data from 2007-2018 $(\mathrm{N}=4297)$

\begin{tabular}{|c|c|c|c|c|c|c|}
\hline \multirow[t]{2}{*}{ Girls } & \multicolumn{4}{|l|}{ Study Year } & \multirow[t]{2}{*}{$P$-for-Trend ${ }^{\mathrm{a}}$} & \multirow[t]{2}{*}{ P-for-Trend ${ }^{b}$} \\
\hline & 2007-2009 & $2010-2012$ & $2013-2015$ & $2016-2018$ & & \\
\hline \multicolumn{7}{|c|}{ Hypercholesterolemia } \\
\hline $10-19$ years & $8.1 \%$ & $9.5 \%$ & $9.2 \%$ & $12.4 \%$ & 0.010 & 0.019 \\
\hline 10-11 years & $6.1 \%$ & $13.2 \%$ & $10.0 \%$ & $9.9 \%$ & 0.287 & 0.311 \\
\hline $12-15$ years & $7.3 \%$ & $6.1 \%$ & $10.2 \%$ & $11.2 \%$ & 0.020 & 0.020 \\
\hline $16-19$ years & $10.1 \%$ & $10.9 \%$ & $8.3 \%$ & $14.4 \%$ & 0.252 & 0.365 \\
\hline \multicolumn{7}{|c|}{ Hypo-HDL-cholesterolemia } \\
\hline $10-19$ years & $12.4 \%$ & $8.9 \%$ & $7.3 \%$ & $6.9 \%$ & $<0.001$ & $<0.001$ \\
\hline 10-11 years & $11.9 \%$ & $10.6 \%$ & $8.6 \%$ & $6.0 \%$ & 0.022 & 0.014 \\
\hline $12-15$ years & $11.9 \%$ & $10.6 \%$ & $8.4 \%$ & $6.1 \%$ & 0.052 & 0.024 \\
\hline $16-19$ years & $11.0 \%$ & $8.4 \%$ & $5.5 \%$ & $5.3 \%$ & 0.002 & 0.001 \\
\hline \multicolumn{7}{|c|}{ Hyper-non-HDL-cholesterolemia } \\
\hline $10-19$ years & $9.4 \%$ & $8.2 \%$ & $7.7 \%$ & $12.8 \%$ & 0.064 & 0.136 \\
\hline 10-11 years & $9.2 \%$ & $10.6 \%$ & $9.7 \%$ & $11.4 \%$ & 0.541 & 0.640 \\
\hline $12-15$ years & $8.0 \%$ & $6.1 \%$ & $8.1 \%$ & $11.6 \%$ & 0.074 & 0.090 \\
\hline $16-19$ years & $11.3 \%$ & $9.1 \%$ & $6.7 \%$ & $14.3 \%$ & 0.379 & 0.603 \\
\hline \multicolumn{7}{|c|}{ Hypertriglyceridemia } \\
\hline $10-19$ years & $14.5 \%$ & $12.1 \%$ & $10.9 \%$ & $14.7 \%$ & 0.895 & 0.799 \\
\hline $10-11$ years & $22.3 \%$ & $21.3 \%$ & $15.8 \%$ & $19.5 \%$ & 0.306 & 0.208 \\
\hline $12-15$ years & $16.5 \%$ & $10.9 \%$ & $10.8 \%$ & $16.6 \%$ & 0.936 & 0.873 \\
\hline $16-19$ years & $9.2 \%$ & $9.6 \%$ & $8.8 \%$ & $11.1 \%$ & 0.486 & 0.499 \\
\hline \multicolumn{7}{|c|}{ Hyper-LDL-cholesterolemia } \\
\hline $10-19$ years & $6.6 \%$ & $6.8 \%$ & $6.7 \%$ & $9.7 \%$ & 0.035 & 0.074 \\
\hline $10-11$ years & $3.9 \%$ & $8.4 \%$ & $4.9 \%$ & $8.4 \%$ & 0.163 & 0.191 \\
\hline $12-15$ years & $5.5 \%$ & $4.7 \%$ & $7.9 \%$ & $8.2 \%$ & 0.050 & 0.055 \\
\hline $16-19$ years & $9.1 \%$ & $8.0 \%$ & $6.5 \%$ & $11.6 \%$ & 0.421 & 0.640 \\
\hline \multicolumn{7}{|l|}{ Dyslipidemia } \\
\hline $10-19$ years & $28.9 \%$ & $25.5 \%$ & $23.9 \%$ & $28.2 \%$ & 0.812 & 0.767 \\
\hline $10-11$ years & $31.2 \%$ & $39.0 \%$ & $29.3 \%$ & $31.9 \%$ & 0.654 & 0.632 \\
\hline $12-15$ years & $29.6 \%$ & $21.3 \%$ & $26.2 \%$ & $31.1 \%$ & 0.462 & 0.467 \\
\hline $16-19$ years & $26.7 \%$ & $24.2 \%$ & $20.8 \%$ & $25.7 \%$ & 0.576 & 0.523 \\
\hline
\end{tabular}

Notes: Values are represented as the number (\%). The reference data were obtained from the 2007-2009 nationwide survey. The prevalence was adjusted for age. ${ }^{a}$ Adjusted for age. ${ }^{b}$ Adjusted for age, BMI, and waist circumference.

Abbreviations: HDL, high-density lipoprotein; LDL, low-density lipoprotein.

$9.8 \%$ to $16.3 \%$ ) and aged $16-19$ years (from $4.5 \%$ to $10.2 \%$ ); statistical significance was found only in boys aged 16-19 years. Decreasing trends in the prevalence of hypo-HDL-cholesterolemia were observed in all age groups; a relatively large decline in the prevalence of hypo-HDL-cholesterolemia was noted in boys aged 1219 years (8.2-8.4\% drop) compared to boys aged 10-11 years $(2.1 \%$ drop). The prevalence of hyper-non-HDLcholesterolemia showed a statistically insignificant increasing trend. There was a significant increase in the prevalence of hyper-LDL-cholesterolemia in boys aged
10-11 years (from $6.2 \%$ to $13.7 \%$ ) and $16-19$ years (from $3.9 \%$ to $8.2 \%$ ); statistical significance remained after adjusting for BMI and waist circumference. Finally, there was a non-significant increase in the prevalence of dyslipidemia from $28.1 \%$ to $34.2 \%$ in boys aged $10-11$ years, but it declined in boys aged 12-19 years; statistical significance was observed only in boys aged 12-15 years (from $31.5 \%$ to $25.4 \%$ ).

In girls, an increasing trend in hypercholesterolemia prevalence was observed in all age groups, but statistical significance was found only in girls aged 12-15 years 

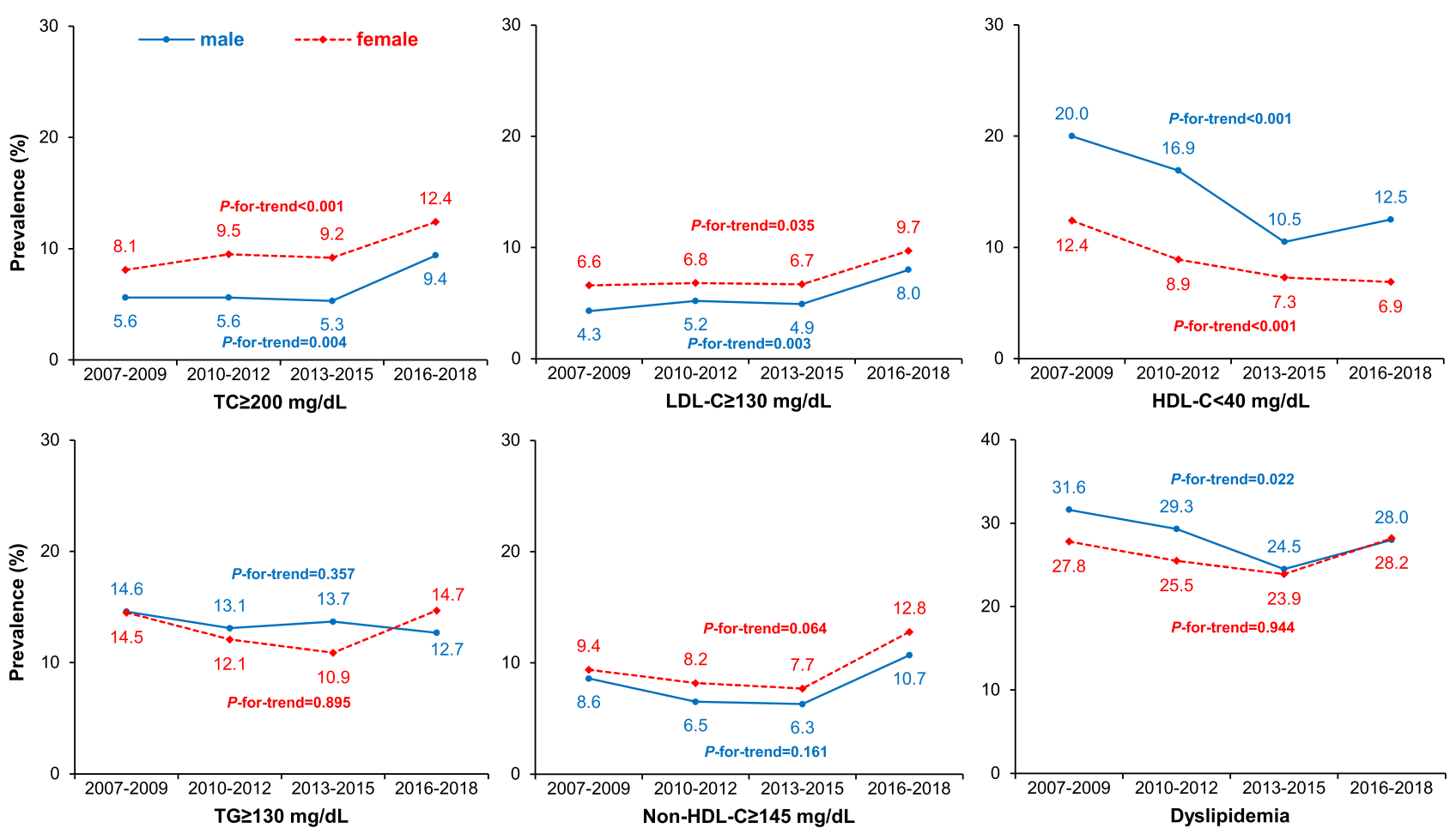

Figure 2 Trends in prevalence of adverse lipid profiles in Korean adolescents from the nationwide survey data from 2007-2018. Note: The prevalence and $\mathrm{P}$-values were adjusted for age.

Abbreviations: TC, total cholesterol; HDL-C, high-density lipoprotein cholesterol; Non-HDL-C, non-high-density lipoprotein cholesterol; TG, triglycerides; LDL-C, lowdensity lipoprotein cholesterol.

(from $7.3 \%$ to $11.2 \%$ ). Decreasing trends in the prevalence of hypo-HDL-cholesterolemia were observed in all age groups, and the decline was identical across age groups (5.7-5.9\% drop). Despite the decrease in hypo-HDLcholesterolemia, the prevalence of hyper-non-HDLcholesterolemia showed a statistically insignificant increasing trend. Similarly, hyper-LDL-cholesterolemia prevalence in girls of all ages showed a rising trend without statistical significance. Lastly, the prevalence of dyslipidemia showed a steady trend in girls of all age groups.

\section{Discussion}

This study shows increasing trends in TC, LDL-C, and non-HDL-C among Korean adolescents between 2007 and 2018. This is the first study to show increasing trends in TC, LDL-C, and non-HDL-C among Korean adolescents between 2007 and 2018. Although the overall dyslipidemia prevalence remained unchanged or decreased due to a substantial decrease in hypo-HDL-cholesterolemia, the prevalence of hypercholesterolemia and hyper-LDLcholesterolemia increased considerably in both sexes. These trends remained statistically significant after adjusting for adiposity, suggesting that other factors might have affected these trends other than increased adiposity.

Our findings, showing adverse trends in TC, LDL-C, and non-HDL-C, are consistent with recent reports from other East Asian countries. Among Japanese adolescents, serum LDL-C levels were significantly increased without a difference in the prevalence of adverse lipid profiles. ${ }^{15} \mathrm{In}$ China, where the prevalence of pediatric obesity has increased, all lipid profiles, including TC, LDL-C, HDL$\mathrm{C}$, and TG, showed adverse trends over the past decade. ${ }^{16}$ In contrast, favorable trends in serum lipid profiles have recently been reported among the pediatric population in the US and Iran. ${ }^{6,17}$

We also found that the prevalence of hypercholesterolemia and hyper-LDL-cholesterolemia in Korean boys and girls almost doubled and reached $9.4-12.4 \%$ and 8.0-9.7\%, respectively, in 2016-2018. Although the age differences limit exact comparisons, this prevalence is much higher than the hyper-LDL-cholesterolemia prevalence of $2.4 \%$ in Japanese boys aged 13 years, ${ }^{15} 5.0-6.1 \%$ in Chinese adolescents, ${ }^{16} 6.4 \%$ in US adolescents, ${ }^{6}$ and $6.5 \%$ in the Iranian pediatric population. ${ }^{17,18}$ Our findings 
are consistent with a recent study showing a marked rise of hypercholesterolemia from $8.8 \%$ in 2007 to $18.0 \%$ in 2018 among Korean adults. ${ }^{19}$ Moreover, a significant increase in the prevalence of hyper-LDL-cholesterolemia (from $15.5 \%$ in 2013 to $19.2 \%$ in 2018 ) has been reported in the Korean population. ${ }^{20,21}$ Since hypercholesterolemia and hyper-LDL-cholesterolemia are well-established risk factors for CVD, our findings highlight the need for public education and close monitoring of Korean CVD incidence and risk factors. ${ }^{14,22}$

Although there was an increasing trend in BMI in both sexes in this study, we postulate that the increase in BMI or waist circumference was not the primary explanation for the unfavorable trends of TC and LDL-C in this study. Adverse trends in hypercholesterolemia and hyper-LDLcholesterolemia maintained their statistical significance even after adjusting for obesity indices. The rise in hypercholesterolemia and hyper-LDL-cholesterolemia was not limited to boys aged 12-19 years with increased central obesity prevalence and was even more remarkable in boys aged 10 11 years with no significant difference in obesity prevalence. Moreover, adverse trends in hypercholesterolemia and hyper-LDL-cholesterolemia were also noted among girls whose obesity prevalence remained constant. These findings suggest that factors other than obesity might have affected these trends in TC and LDL-C. Further studies, including those that evaluate lifestyle and dietary factors, are needed to elucidate the causes of these trends.

Obesity is a well-known risk factor for hypertriglyceridemia and hypo-HDL-cholesterolemia in adolescents and adults, ${ }^{23}$ and intensive lifestyle modifications, including moderate caloric restriction and regular aerobic physical activity, are recommended in obese children and adolescents. $^{24}$ Despite the increase in BMI among Korean adolescents, TG levels remained unchanged, and HDL-C levels showed increasing trends in this study. Notably, the prevalence of hypo-HDL-cholesterolemia almost halved in both sexes, and the decline was more prominent in boys than in girls. Similar to our findings, a recent study demonstrated increasing trends in serum HDL-C among Korean adults between 2005 and 2015 despite a significant increase in BMI in Korean men. ${ }^{19}$ Another recent study found that the intake of total fat and saturated/unsaturated fatty acids steadily increased among Korean adults from 2007 to $2015 .^{25}$ A survey of the longterm national trends in lipid profiles also suggested that an increasing trend in TC and HDL-C levels was accompanied by a rise in dietary saturated fat intake in the
Japanese population. ${ }^{26}$ These previous studies provide new insights into the trends in lipid profiles in the Korean population. Saturated fat intake has been reported to raise serum TC and LDL-C levels in multiple randomized controlled trials $;{ }^{27}$ therefore, international guidelines for the management of dyslipidemia recommend that saturated fat consumption should be less than $10 \%$ of total caloric intake and further lower in individuals with hypercholesterolemia. ${ }^{28}$ Intake of most fatty acid types (except for trans-fatty acids) has been shown to increase HDL-C levels, possibly by increasing the transport rates and decreasing the fractional catabolic rates of HDL cholesterol ester and apolipoprotein A-I. ${ }^{27,29}$ In addition, a recent study observed that the consumption of dairy products was associated with higher TC, LDL$\mathrm{C}$, and HDL-C levels in patients with dyslipidemia. ${ }^{30}$ Owing to the lack of data on dietary consumption in KNHANES, especially in adolescents aged 10-11 years, our study could not determine the possible association between the changes in lipid profiles and nutritional factors. However, a simultaneous increase in TC, HDL-C, and LDL-C levels in Korean adolescents suggests a possible role of changes in dietary fat or dairy consumption in this population; further studies are warranted in the future.

Traditionally, HDL-C has been considered "a good cholesterol" owing to its pivotal role in the reverse cholesterol transport of HDL particles. ${ }^{31}$ However, it cannot be presumed that the increase in HDL-C will have a protective effect on CVD risk in the Korean population, given the increase in TC and LDL-C levels. HDL-C levels indicate "the amount of cholesterol" carried by HDL particles, not the "atheroprotective functionality" of HDL particles. ${ }^{32}$ Multiple randomized controlled studies demonstrated that increased HDL-C levels after treatment with cholesterol ester transfer inhibitors could not reduce ASCVD events. ${ }^{33}$ Recent prospective cohort studies have also shown that the relationship between HDL-C and CVD is U-shaped, ${ }^{34,35}$ not an inverse linear association. The mechanism remains uncertain, although it might be partly explained by a disparity between the HDL-C concentration and the cardioprotective function of HDL particles. ${ }^{36}$

Further, non-HDL-C, a crucial predictor for CVD, has increased in both sexes, despite the overall increase in HDL$\mathrm{C}$ among the Korean pediatric population. Although the prevalence of dyslipidemia slightly decreased in boys aged 12-19 years due to a significant decline in hypo-HDLcholesterolemia, there was a non-significant increase in its 
prevalence in boys aged 10-11 years and was unchanged in girls. Therefore, to elucidate the impact of these trends in lipid profiles in Korean populations, further research on the changes in the characteristics of lipoprotein particle profiles, including their structure and function, and longitudinal studies including CVD incidence are needed.

A notable limitation of this study is that we did not assess detailed comorbidity data and lifestyle factors associated with lipid levels, such as diet patterns, physical activity, and smoking status, because of the lack of this information in the KNHANES dataset among adolescents aged 10-11 years. Secular trends in these factors might have influenced the changes in lipid profiles. Nevertheless, this study reports the most recent trends in serum lipid concentrations and the prevalence of abnormal lipid profiles in Korean adolescents by sex and age group using nationally representative data.

\section{Conclusion}

In conclusion, the results of this study indicate a remarkable increase in the prevalence of hyper-LDLcholesterolemia and hypercholesterolemia and a significant decline in the prevalence of hypo-HDLcholesterolemia among Korean adolescents between 2007 and 2018. Our results highlight the need for ongoing monitoring of the serum lipid profiles in Korean adolescents and further studies on casual factors for these trends.

\section{Acknowledgments}

We thank the Korea Disease Control and Prevention Agency for enabling us to utilize the KNHANES data.

\section{Disclosure}

The authors report no conflicts of interest in this work.

\section{References}

1. Khan MA, Hashim MJ, Mustafa H, et al. Global epidemiology of ischemic heart disease: results from the Global Burden of Disease Study. Cureus. 2020;12:e9349.

2. Shin JH, Cheong JI, Cheuh HW, Yoo JH. Limitations of current screening methods for lipid disorders in Korean adolescents and a proposal for an effective detection method: a nationwide, cross-sectional study. Ann Pediatr Endocrinol Metab. 2020;25: 265-271. doi:10.6065/apem.2040098.049

3. Frontini MG, Srinivasan SR, Xu J, Tang R, Bond MG, Berenson GS. Usefulness of childhood non-high density lipoprotein cholesterol levels versus other lipoprotein measures in predicting adult subclinical atherosclerosis: the Bogalusa Heart Study. Pediatrics. 2008;121: 924-929. doi:10.1542/peds.2007-1472

4. Kim JH, Moon JS. Secular trends in pediatric overweight and obesity in Korea. J Obes Metab Syndr. 2020;29:12-17. doi:10.7570/jomes20002
5. Kim SH, Ahn BC, Joung H, Park MJ. Lipid profiles and prevalence of dyslipidemia in Korean adolescents. Endocrinol Metab. 2012;27:208-216. doi:10.3803/EnM.2012.27.3.208

6. Perak AM, Ning H, Kit BK, et al. Trends in levels of lipids and apolipoprotein B in US youths aged 6 to 19 years, 1999-2016. JAMA. 2019;321:1895-1905. doi:10.1001/jama.2019.4984

7. Kim SH, Song YH, Park S, Park MJ. Impact of lifestyle factors on trends in lipid profiles among Korean adolescents: the Korea National Health and Nutrition Examination Surveys study, 1998 and 2010. Korean J Pediatr. 2016;59:65-73. doi:10.3345/kjp.2016.59.2.65

8. World Health Organization. The Asia-Pacific Perspective: Redefining Obesity and Its Treatment. Sydney: Health Communications Australia; 2000:15-18.

9. Kim JH, Yun S, Hwang SS, et al. The 2017 Korean National Growth Charts for children and adolescents: development, improvement, and prospects. Korean $J$ Pediatr. 2018;61:135-149. doi:10.3345/ kjp.2018.61.5.135

10. Moon JS, Lee SY, Nam CM, et al. 2007 Korean National Growth Charts: review of developmental process and an outlook. Korean J Pediatr. 2008;51:1-25. doi:10.3345/kjp.2008.51.1.1

11. Lee SY, Park HS, Kim DJ, et al. Appropriate waist circumference cutoff points for central obesity in Korean adults. Diabetes Res Clin Pract. 2007;75:72-80. doi:10.1016/j.diabres.2006.04.013

12. Friedewald WT, Levy RI, Fredrickson DS. Estimation of the concentration of low-density lipoprotein cholesterol in plasma, without use of the preparative ultracentrifuge. Clin Chem. 1972;18:499-502. doi:10.1093/clinchem/18.6.499

13. National Heart, Lung, and Blood Institute. Expert panel on integrated guidelines for cardiovascular health and risk reduction in children and adolescents: summary report. Pediatrics. 2011;128:S213-S256.

14. Lim JS, Kim EY, Kim JH, et al. 2017 Clinical practice guidelines for dyslipidemia of Korean children and adolescents. Ann Pediatr Endocrinol Metab. 2020;25:199-207. doi:10.6065/apem.2040198.099

15. Ding W, Cheng H, Yan Y, et al. 10-year trends in serum lipid levels and dyslipidemia among children and adolescents from several schools in Beijing, China. $J$ Epidemiol. 2016;26:637-645. doi:10.2188/jea.JE20140252

16. Habib A, Molayemat M, Habib A. Association of lipid profile and BMI Z-score in southern Iranian children and adolescents. J Pediatr Endocrinol Metab. 2019;32:827-835. doi:10.1515/jpem-2019-0002

17. Baghbani-Oskouei A, Tohidi M, Asgari S, Ramezankhani A, Azizi F, Hadaegh F. Serum lipids during 20 years in the Tehran lipid and glucose study: prevalence, trends and impact on non-communicable diseases. Int $J$ Endocrinol Metab. 2018;16:e84750. doi:10.5812/ ijem. 84750

18. Ha KH, Kwon HS, Kim DJ. Epidemiologic characteristics of dyslipidemia in Korea. J Lipid Atheroscler. 2015;4:93-99. doi:10.12997/ jla.2015.4.2.93

19. Kwon YJ, Lee JW, Kang HT. Secular trends in lipid profiles in Korean adults based on the 2005-2015 KNHANES. Int J Environ Res Public Health. 2019;16:2555. doi:10.3390/ijerph16142555

20. The Korean Society of Lipid and Atherosclerosis. Dyslipidemia fact sheet in Korea; 2020. Available from: https://www.lipid.or.kr/bbs/ skin/default/download.php?code=fact_sheet\&number=1391. Accessed June 22, 2021.

21. Jellinger PS, Smith DA, Mehta AE, et al. AACE task force for the management of dyslipidemia and prevention of atherosclerosis writing committee. Endocr Pract. 2012;18:270-293.

22. Kouda K, Iki M, Fujita Y, et al. Trends in serum lipid levels of a 10and 13-year-old population in Fukuroi city, Japan (2007-2017). J Epidemiol. 2020;30:24-29. doi:10.2188/jea.JE20180164

23. Chu SY, Jung JH, Park MJ, Kim SH. Risk assessment of metabolic syndrome in adolescents using the triglyceride/high-density lipoprotein cholesterol ratio and the total cholesterol/high-density lipoprotein cholesterol ratio. Ann Pediatr Endocrinol Metab. 2019;24:41-48. doi:10.6065/apem.2019.24.1.41 
24. Grundy SM, Stone NJ, Bailey AL, et al. 2018 AHA/ACC/AACVPR/ AAPA/ABC/ACPM/ADA/AGS/APhA/ASPC/NLA/PCNA guideline on the management of blood cholesterol: a report of the American College of Cardiology/American Heart Association Task Force on Clinical Practice Guidelines [published correction appears in Circulation. 2019 Jun 18;139(25):e1182-e1186]. Circulation. 2019;139(25):e1082-e1143.

25. Song S, Shim JE. Trends in dietary intake of total fat and fatty acids among Korean adolescents from 2007 to 2017. Nutrients. 2019;11:3073. doi:10.3390/nu11123073

26. NCD Risk Factor Collaboration (NCD-RisC). National trends in total cholesterol obscure heterogeneous changes in HDL and non-HDL cholesterol and total-to-HDL cholesterol ratio: a pooled analysis of 458 population-based studies in Asian and Western countries. Int J Epidemiol. 2020;49:173-192. doi:10.1093/ije/dyz099

27. Mensink RP, Zock PL, Kester AD, Katan MB. Effects of dietary fatty acids and carbohydrates on the ratio of serum total to HDL cholesterol and on serum lipids and apolipoproteins: a meta-analysis of 60 controlled trials. Am J Clin Nutr. 2003;77:1146-1155. doi:10.1093/ajen/77.5.1146

28. Mach F, Baigent C, Catapano AL, et al. 2019 ESC/EAS Guidelines for the management of dyslipidaemias: lipid modification to reduce cardiovascular risk [published correction appears in Eur Heart J. 2020 Nov 21;41(44):4255]. Eur Heart J. 2020;41(1):111-188.

29. Hayek T, Ito Y, Azrolan N, et al. Dietary fat increases high density lipoprotein (HDL) levels both by increasing the transport rates and decreasing the fractional catabolic rates of HDL cholesterol ester and apolipoprotein (Apo) A-I. Presentation of a new animal model and mechanistic studies in human Apo A-I transgenic and control mice. J Clin Invest. 1993;91:1665-1671.
30. Formisano E, Pasta A, Cremonini AL, Di Lorenzo I, Sukkar SG, Pisciotta L. Effects of a mediterranean diet, dairy, and meat products on different phenotypes of dyslipidemia: a preliminary retrospective analysis. Nutrients. 2021;13(4):1161. doi:10.3390/nu130 41161

31. Katsa ME, Ioannidis A, Sachlas A, Dimopoulos I, Chatzipanagiotou S, Rojas Gil AP. The roles of triglyceride/high-density lipoprotein cholesterol ratio and uric acid as predisposing factors for metabolic syndrome in healthy children. Ann Pediatr Endocrinol Metab. 2019;24:172-179. doi:10.6065/apem.2019.24. 3.172

32. Ronsein GE, Heinecke JW. Time to ditch HDL-C as a measure of HDL function? Curr Opin Lipidol. 2017;28:414-418. doi:10.1097/ MOL.0000000000000446

33. Tall AR, Rader DJ. Trials and tribulations of CETP inhibitors. Circ Res. 2018;122:106-112. doi:10.1161/CIRCRESAHA.117. 311978

34. Madsen CM, Varbo A, Nordestgaard BG. Extreme high high-density lipoprotein cholesterol is paradoxically associated with high mortality in men and women: two prospective cohort studies. Eur Heart $J$. 2017;38:2478-2486. doi:10.1093/eurheartj/ehx163

35. Ko DT, Alter DA, Guo H, et al. High-density lipoprotein cholesterol and cause-specific mortality in individuals without previous cardiovascular conditions: the CANHEART Study. J Am Coll Cardiol. 2016;68:2073-2083. doi:10.1016/j.jacc.2016.08.038

36. Rader DJ, Tall AR. The not-so-simple HDL story: is it time to revise the HDL cholesterol hypothesis? Nat Med. 2012;18:1344-1346. doi:10.1038/nm.2937

\section{Publish your work in this journal}

Diabetes, Metabolic Syndrome and Obesity: Targets and Therapy is an international, peer-reviewed open-access journal committed to the rapid publication of the latest laboratory and clinical findings in the fields of diabetes, metabolic syndrome and obesity research. Original research, review, case reports, hypothesis formation, expert opinion and commentaries are all considered for publication. The manuscript management system is completely online and includes a very quick and fair peer-review system, which is all easy to use. Visit http://www.dovepress.com/testimonials.php to read real quotes from published authors. 